non-traumatic acute pain, between 2014 and 2015. All included registries must had a radiological assessment. The gathering of registries concluded after reach $120 \%$ of the estimated sample size for a non finite theoretical population, a precision of $3 \%$ and a hypothetical estimated prevalence of $7 \%$ based on local previous studies of prevalence of vertebral osteoporotic fractures. Vertebral body measures were performed according to Genant scale recommendations from D7 to $L 5$ as far as possible according to the field of study of the radiological chart plate.

Results: 275 randomized registries of dorsal and lumbar pain were included (total=550). Among patients with dorsal pain we identified $62(22.5 \%), 30(10.9 \%)$ and $18(6.5 \%)$ vertebral deformities grade I, II and III respectively. Among patients with lumbar pain we identified $31(11.2 \%), 49(17.8 \%)$ and $33(12 \%)$ vertebral deformities grade I, II and III respectively. Prevalence of any grade of dorsal vertebral deformity was $40.00 \%(\mathrm{Cl} 34.39-45.89)$ and lumbar was $41.09 \%(\mathrm{Cl}$ $95 \% 35.44-46.99$ ). Lumbar vertebral body deformities grade I and II summed $70.7 \%$ while dorsal grade I and II summed $83.6 \%$. From the 93 vertebral body deformities grade I, $6.4 \%$ were recognized in their clinical histories, $20.2 \%$ of the grade II deformities and $92.1 \%$ of the grade III deformities, $(P<0.001)$.

Conclusions: Although our population sample is circumscribed to symptomatic patients, our results contribute with prevalence of vertebral body deformities in postmenopausal patients grade I and II and who were mostly unnoticed. Proper identification of vertebral body deformities in patients with osteoporosis is crucial to decide treatment strategies in patients with known osteoporosis. Due to that, prevalence studies of this kind are relevant and useful to avoid diagnostic mistakes. Disclosure of Interest: None declared

DOI: 10.1136/annrheumdis-2017-eular.5605

\section{FRI0543 WHICH FACTORS CAN HELP PREDICT FRAGILITY FRACTURES IN PATIENTS DIAGNOSED WITH INFLAMMATORY BOWEL DISEASE? A CASE-CONTROL STUDY}

D. Ahmed ${ }^{1}$, M. Bukhari ${ }^{2} .{ }^{1}$ Lancaster Medical School; ${ }^{2}$ Rheumatology, University Hospitals of Morecambe Bay NHS Trust, Lancaster, United Kingdom

Background: Inflammatory Bowel Disease (IBD) is a known risk factor for developing low bone mineral density (BMD) osteoporosis, due to malabsorption and treatment with steroids. These patients are more susceptible to fragility fractures. Though the percentage of such fractures is low, they can be associated with impaired mortality and morbidity. The difficulty lies in early detection of patients at an increased risk of fractures. Currently the diagnosis of osteoporosis and predictions of fracture risk are calculated assessing patient BMD on dual energy X-ray absorptiometry (DEXA). However, previous studies suggest that despite a decreasing BMD being significantly associated with an increased risk of fracture, its measurement alone is fairly restricted in predicting them; other patient factors must also be brought into consideration (1).

Objectives: To identify specific factors which may assist in the prediction of fragility fractures in a cohort of patients diagnosed with IBD.

Methods: Patients referred to a DEXA scan in the North West of England were identified and those with a referral reason of IBD were studied. Factors measured at BMD scanning include patient age, height, weight, lumbar and femoral head bmd, BMI, smoking history, alcohol use, family history of fractures, steroid exposure, rheumatoid arthritis and secondary osteoporosis. Patients where assorted into cases and controls after adjusting for age and gender. They were then analysed using $T$ tests for continuous variables and Chi squared tests for categorical variables. Univariate and multivariate logical regression models were then utilised to identify factors predicting fractures

Results: 938 patients were identified of which $721(76.9 \%)$ were female with an average age of 58 as compared to an average age of 53 in men. 274 patients (29\%) had fractures of which 238 were females $(87 \%)$, at an average age of 63 compared to 60 in men. Men were shown to have a greater risk of fractures. Results of the univariate analysis are shown below.

\begin{tabular}{lccccc}
\hline Predictor & \multicolumn{3}{l}{ All Pts Pts with Fracture Pts without Fracture P-value } & $\begin{array}{c}\text { Odds ratio } \\
(95 \% \mathrm{Cl})\end{array}$ \\
\hline Age at scan & 56.8 & 62.5 & 54.5 & 0.00 & $1.03(1.03-1.05)$ \\
Height & 163.7 & 161.2 & 164.7 & 0.21 & $0.99(0.96-1.01)$ \\
Weight & 71.6 & 70.0 & 72.2 & 0.63 & $0.99(0.99-1.08)$ \\
Alcohol & 52 & 16 & 36 & 0.24 & $1.48(0.77-2.82)$ \\
Smoking & 373 & 109 & 264 & 0.69 & $1.06(0.79-1.44)$ \\
Family History & 169 & 53 & 116 & 0.34 & $1.19(0.81-1.74)$ \\
RA & 49 & 20 & 29 & 0.20 & $1.49(0.81-2.75)$ \\
Secondary op & 108 & 40 & 78 & 0.93 & $1.02(0.66-1.56)$ \\
Left Femoral Neck BMD & 0.87 & 0.82 & 0.89 & 0.00 & $0.11(0.04-0.31)$ \\
Right Femoral Neck BMD & 0.92 & 0.86 & 0.94 & 0.00 & $0.19(0.62-0.58)$ \\
Lumbar Spine BMD & 1.22 & 1.01 & 1.10 & 0.00 & $0.10(0.44-0.24)$ \\
BMI & 28.7 & 26.9 & 26.6 & 0.96 & $0.99(0.97-1.03)$ \\
Steroid & 617 & 134 & 483 & 0.00 & $0.49(0.36-0.67)$ \\
\hline
\end{tabular}

In the multivariate analysis, statistically significant variables were BMI (OR 1.05, $95 \% \mathrm{Cl} 1.01-1.08$ ) and steroids (OR $0.49,95 \% \mathrm{Cl} 0.35-0.69$ ) with steroids being protective against fractures.

Conclusions: In the univariate analysis several risk factors are shown to be associated with fractures. These include femoral neck BMD, steroid use, lumbar BMD and the patient age at the time of the scan. The multivariate analysis showed that the biggest predictor after adjusting for age and gender is steroids and BMI with steroids being protective.

References:

[1] Marshall D, Johnell $\mathrm{O}$, Wedel $\mathrm{H}$. Meta-analysis of how well measures of bone mineral density predict occurrence of osteoporotic fractures. BMJ. 1996;312(7041):1254-9.

Disclosure of Interest: None declared

DOI: 10.1136/annrheumdis-2017-eular.4013

\section{FRI0544 CLINICAL AND DEMOGRAPHIC PROFILE OF PATIENTS CONSULTING FOR FRAGILITY FRACTURES IN A HOSPITAL IN COLOMBIA DURING THE YEARS 2014-2016: IMAGE OF THE COLOMBIAN HEALTH SYSTEM}

D.G. Fernández-Ávila, D.N. Rincón-Riaño, D. Pinzón, J.M. Gutiérrez. Rheumatology, Hospital Universitario San Ignacio - Pontificia Universidad Javeriana, Bogotá, Colombia

Background: Osteoporosis represents a major public health problem due to the seriousness of a patient's main complication: fracture. The lack of the Colombian health system for a structured public policy aiming diagnosis and early intervention generates a high volume of patients having fragility fractures.

Objectives: To describe the clinical and demographic characteristics of patients with fragility fractures seen in our hospital. To describe the risk factors for fragility fractures. To inquire about the patient's knowledge about osteoporosis. To follow each case establishing whether after presenting the complication (fracture), the patient would receive an ambulatory treatment covered by the health insurance. Methods: Cross-sectional descriptive study

Results: 111 patients mean age of 74.4 years ( \pm 11.3 years), 84 (75.6\%) were women. All consulted for osteoporotic fracture. The most frequent type of fracture was hip $(51.4 \%)$, followed by vertebra $(23.4 \%)$, wrist $(22.5 \%)$ and humerus (4.5\%). $87.4 \%(n=97)$ had no personal history of fracture and only $1 \%$ had a history of frailty fracture in a first-degree relative. Risk factors: $7.2 \%(n=8)$ used glucocorticoids, $3.6 \%(n=4)$ antiepileptics and $3.6 \%(n=4)$ warfarin. $21.6 \%(n=24)$ were smokers. $77.5 \%(n=86)$ had never previously undergone a densitometry despite the fact that, because of their age, they had indicated that this study had previously been performed. Knowledge of osteoporosis by patients: $49.5 \%$ $(n=55)$ did not know that osteoporosis was present, 58.6\% $(n=65)$ did not know that fracture was the main complication of this disease and $62.2 \%=69$ ) does not relate to fractures with osteoporosis. All patients were educated and sensitized about osteoporosis and the importance of diagnosis and treatment and they were given an order to perform densitometry at discharge, despite the above $24.3 \%(n=27)$ densitometry was performed in the next year of the fracture. As for treatment, $33.3 \%(n=37)$ received calcium plus vitamin D. Only $9.9 \%(n=11)$ received treatment for osteoporosis (7 patients with bisphosphonate and 4 with denosumab), none received teriparatide osteoformer therapy.

Conclusions: The present study demonstrates the lack of understanding by the Colombian patients about osteoporosis. Despite of clear indications described international guidelines, we have found a the lack of densitometry measurements on our follow up patients. More serious, only $10 \%$ of the patients received treatment for osteoporosis and none of them used a osteoformer therapy. This proves the suboptimal follow-up made by the health insurance companies of our country. Urgent educational and public health policies are needed.

Disclosure of Interest: None declared

DOI: 10.1136/annrheumdis-2017-eular.5625

\section{FRI0545 COMPARISON OF MINERAL BONE DENSITY IN HIV-INFECTED PATIENTS FOLLOWED IN A SPANISH TERTIARY HOSPITAL WITH THAT OF NON HIV-INFECTED SPANISH POPULATION}

F. López Gutiérrez ${ }^{1}$, W.A. Sifuentes Giraldo ${ }^{1}$, J.L. Casado Osorio ${ }^{2}$, M. Vázquez Díaz ${ }^{1} .{ }^{1}$ Rheumatology; ${ }^{2}$ Infectious Diseases, Ramon y Cajal University Hospital, Madrid, Spain

Background: Patients with human immunodeficiency virus (HIV) have a higher prevalence of low bone mineral density (BMD) and fractures than the general population, but there are no comparative studies in Spanish population.

Objectives: To assess the BMD in HIV-infected patients followed in a tertiary hospital of Madrid and compare it with the ESOSVAL cohort, which included 11035 patients and is representative of non-HIV population seen in Spanish tertiary centers.

Methods: We performed a cross-sectional study in which BMD values were determined in a prospective cohort that included HIV-infected patients seen our center during the period 2010-2015. Collected data included demography, comorbidities, treatment and densitometric variables.

Results: 93 patients from a total of a total of 924 with BMD data were eligible for the study after discarding those younger than 55 years, because that group is not included in the ESOSVAL cohort. Mean age of patients of our whole cohort was 43.8 years (range: $17-83$ ), $11 \%$ were older than 55 years, of whom 83 were men $(83 \%)$. Most of them were Caucasians, with a mean body mass index 24.1 (range: 14,7-40.6). Median time of HIV infection was 162,6 months (interquartile range [IQR]: 77.7- 283,3), median CD4+ cells nadir was 224 (IQR: 100-332) and median maximun viral load was 4,9 log (IQR: 4,3-5,4); concomitant hepatitis C 